\title{
APORTACIÓN AL CONOCIMIENTO DEL TEXTO DE LA HISTORIA DE LOS ANIMALES DE ELIANO Y ASUNTOS CONEXOS
}

\begin{abstract}
The author gives some examples in order to demonstrate that Aelian took most of his stories in Historia Animalium from previous works, especially from the Historia Animalium by Aristotle, the De sollertia animalium by Plutarch, the Naturalis Historia by Pliny and also from works by Oppian and Athenaeus.
\end{abstract}

01. La mayoría de las narraciones de la Historia de los animales de Eliano no es obra inventada por el propio Eliano, sino por autores anteriores de los que él la tomó, como lo demuestra inequivocamente el hecho de que éstas aparecen, a menudo literalmente e incluso en el mismo orden de palabras, en obras más antiguas, singularmente en la Historia de los animales de Aristóteles, en el De sollertia animalium de Plutarco, en la $\mathrm{Na}$ turalis Historia de Plinio, en Opiano y en Ateneo.

02. Este dato (el hecho de que el texto de la Historia de los animales de Eliano aparezca sustancialmente idéntico en los varios autores citados) proporciona a nuestras sugerencias textuales un criterio determinante $y$, de acuerdo con él, cierta dosis de fiabilidad, por cuanto que son susceptibles de ser contrastadas objetivamente y de salir aprobadas o rechazadas de esta prueba, todo lo cual las diferencia esencialmente de las meras conjeturas.

1 Según Perizonius y Wyttenbach, Eliano habria copiado sus historias de Plutarco, y, en cambio, según Wellmann, ambos, Eliano y Plutarco, derivaban, independientemente, de Alejandro de Mindo a través de la obra lexicográfica de Pánfilo. La independencia de Eliano y Plutarco es confirmada por Richmond.

Igualmente, Eliano copió la mayor parte de sus narraciones relativas a los animales de agua de los Halieutica de Opiano, como acertadamente sostenian los antiguos y también Mair en su edición de la obra de Opiano de 1928 y según confirma Keydell, 
1. Eliano, $H A$ VI 15 , y Plinio, $N H$ IX 27 , se refieren, esencialmente en iguales términos, al amor profesado entre un delfin y un joven de la ciudad de Jaso. Sólo difieren en un punto: al final de la citada narración

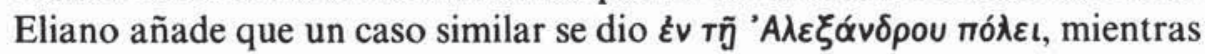
en igual lugar Plinio señala que Alexander Magnus nombró al joven de Jaso sacerdote de Neptuno en Babilonia.

La confrontación del texto de Eliano con el de Plinio sugiere que debe haber, en uno u otro autor, alguna confusión textual, aunque no es fácil intuir quién de los dos conservó el estado de cosas originario y quién lo adulteró. Esto es: no nos es dado verificar si inicialmente la historieta de los amores entre un joven y un delfin era doble (una ocurrida «en Jaso» y otra "en Alejandría», como indica Eliano) o si era una sola pero ligada de una u otra manera a Alejandro (como sostiene Plinio).

2. Eliano, HA VIII 3, y Plutarco, De sollertia animalium $984 \mathrm{~F}-985$ A y B, narran con una identidad total la historieta que cuenta que un tal Cérano de Paros, al ver en la ciudad de Bizancio que unos delfines, tras haber caido en la red, estaban a punto de ser matados, los compró y los dejó libres, acción piadosa a la que posteriormente correspondieron los agradecidos delfines, sacándolo sano y salvo del mar con ocasión del naufragio de la nave pentecóntoro en que el susodicho Cérano navegaba.

Sólo difieren en lo siguiente: según Eliano, la nave pentecóntoro de

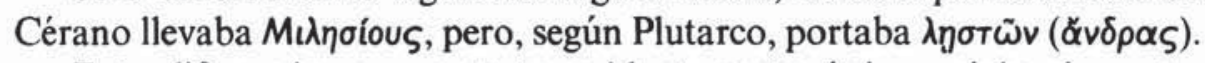

Esta diferencia, en un texto evidentemente único originariamente, pudo haber nacido de un error. Lo originario pudo ser el genitivo plural

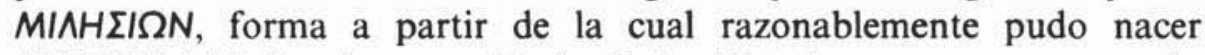
$\Lambda H \Sigma T \Omega N$ (al haber desaparecido la sílaba MI, tal vez por estar separada del resto de la palabra, lo que era factible si $M I$ ocupaba el final de línea y el resto de la palabra el inicial de la línea siguiente). Por esa vía se confundiría $\Lambda H \Sigma I \Omega N$ con $\Lambda H \Sigma T \Omega N$, habida cuenta de su estrecho parecido formal, incrementado por el hecho de que en época de Eliano no se escribía la iota de $\lambda \eta \sigma \tau \tau \tilde{\omega} v^{2}$.

"Oppians Gedicht von der Fischerei und Aelians Tiergeschichte», Hermes 72, 1937, pp. 411-434, aunque algunas de estas historietas las toman ambos, independientemente, del Epitome de Aristófanes.

Pero Eliano tomó su material zoológico no sólo de las fuentes citadas, sino también de compendios y escolios de gramáticos. Cf. sobre esta importante cuestión el excepcional y fundamental trabajo de J. Richmond, Chapters on Greek Fish-Lore, Wiesbaden 1973.

${ }^{2}$ Cf. M. Lejeune, Phonétique historique du mycénien et du grec ancien, Paris 1972, pp. 226-7. 
También cabe que lo originario fuera $M^{\prime} \Lambda H \Sigma T \Omega N^{3}$ (esto es, precedida esta última forma del numeral $M$ ' 'cuarenta'), a partir de lo cual surgi-

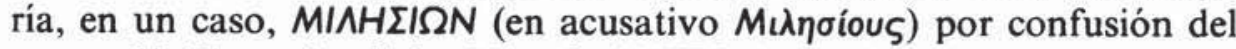
numeral ${ }^{4} M^{\prime}$ con la sílaba $M I$ y de $\Lambda H \Sigma T \Omega N$ con $\Lambda H \Sigma I \Omega N$, y, en el otro, $\Lambda H \Sigma T \Omega N$, por incomprensión u olvido del numeral precedente ${ }^{5}$.

3. Eliano, $H A \mathrm{~V} 3$, al referirse a la protección que procuran los indios

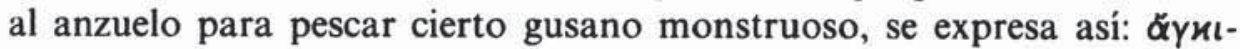

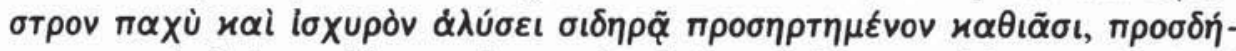

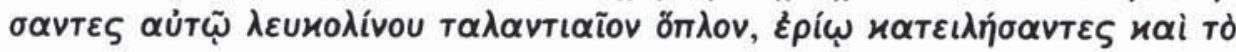
หà тó...

Pasajes de otros autores parecidos al de Eliano son: Diocl., Fr. 142,

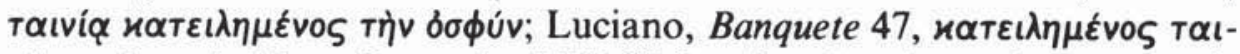

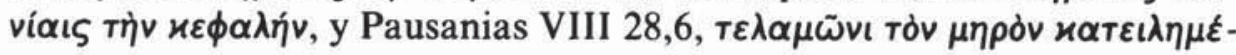
vov.

Por todo ello, cuando Eliano, $H A$ XI 23, se refiere a la raya azafranada que circunda la boca del pez citarodo con la forma $\varkappa \alpha т \varepsilon i \lambda \eta \eta т \alpha \iota$, enten-

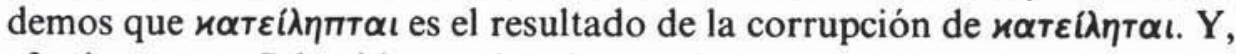
efectivamente, Schneider, en igual proceder que nosotros, corrigió la for-

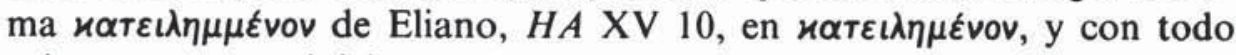
acierto, a nuestro juicio.

4. Plinio, $N H$ VII 13, igual que Eliano, $H A$ XII 39 , sitúa a la estirpe de los «Hijos de serpiente» en la zona del Helesponto, zona que él liga a la ciudad de "Pario del Helesponto", con estas palabras: Crates Pergamenus in Hellesponto, circa Parium, genus hominum fuisse, quos Ophiogenes uocat, serpentium ictus contactu leuare solitos et manu inposita uenena extrahere corpori.

Sin embargo, el propio Plinio, $N H$ XXVIII 30, sitúa a estos mismos individuos «en la isla de Paros», en el siguiente texto: Quorundam hominum tota corpora prosunt, ut ex his familiis quae sunt terrori serpentibus tactu ipso leuant percussos suctuue e tumido, quorum e genere sunt Psylli Marsique et qui Ophiogenes uocantur in insula Paro.

\footnotetext{
3 Que esta sugerencia es razonable puede deducirse de Kronenberg, Mnemosyne 52,1924, p. 101.

4 Cf. casos semejantes en L. Gil, "Notas críticas al texto de Lisias», EMERITA 32, 1964, pp. 35-47.

5 Cf. casos semejantes en R. Renehan, Greek textual criticism, Cambridge, Massachusetts, 1969, pp. 94-5, y la tradición, posiblemente de fondo o trasfondo antiquísimo, que habla de Alí Babá y los cua rent a ladrones.
} 
¿Cómo se explica esta última afirmación de Plinio que contradice la anterior del mismo autor y la de Eliano? Muy sencillamente: la expresión in insula Paro es un error por Pario, ciudad ubicada en la zona del Helesponto de Frigia.

5. Los cuatro autores, Aristóteles, Plutarco, Eliano y Plinio, hablan del vuelo de las grullas y de la dirección que llevan en relación con el viento, utilizando todos ellos la misma fuente, según lo demuestra la coincidencia formal y de contenido de las respectivas narraciones.

Pero difieren en un punto: según Aristóteles y Plutarco las grullas vuelan «contra el viento», y, en cambio, al decir de Eliano y Plinio, vuelan «a favor del viento".

He aquí los textos respectivos.

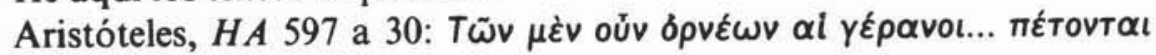
$\delta \dot{\varepsilon}$ прòऽ тò $\pi v \varepsilon \tilde{u} \mu \alpha$.

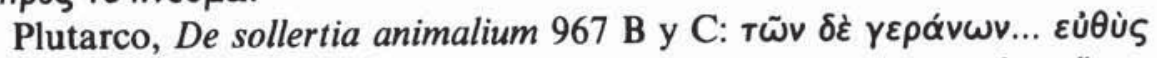

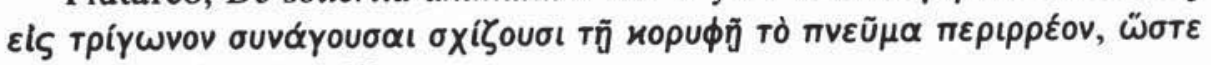

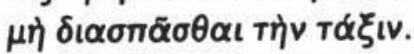

Y el propio Plutarco, en 979 A y C, insiste en lo mismo asi: $\eta \dot{\varepsilon} \delta \varepsilon \varepsilon$ -

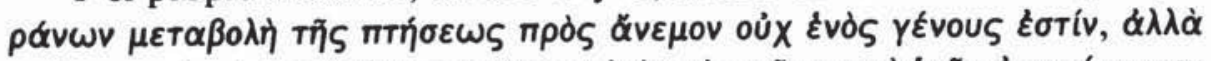

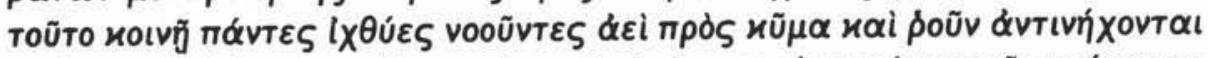

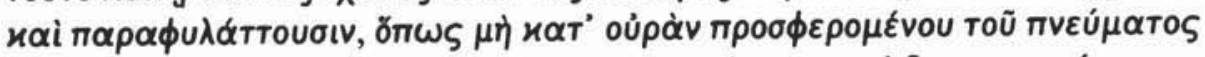

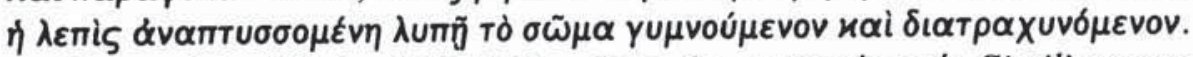

En cambio, Plinio $\mathrm{NH}$ X 63, afirma lo contrario así: Simili anseres quoque et olores ratione commeant, sed horum uolatus cernitur. Liburnicarum more rostrato impetu feruntur, facilius ita findentes aera quam si recta fronte inpellerent; a tergo sensim dilatante se cuneo porrigitur agmen largeque inpellenti praebetur aurae.

En el mismo sentido que Plinio se manifiesta Eliano, $H A$ III 13, así:

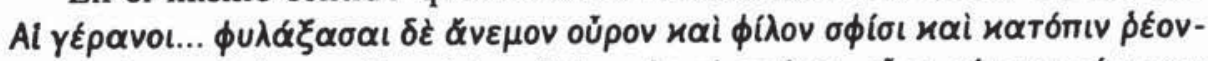

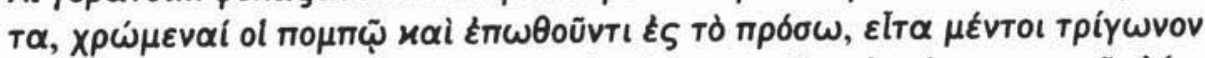

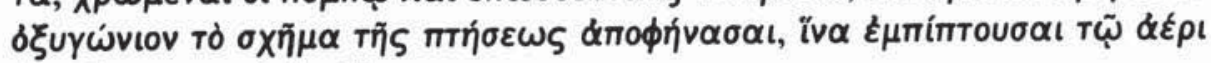

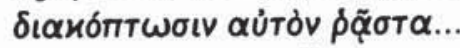

¿Cómo se explica que estos autores incurran en tan flagrante contradicción cuando es claro que todos ellos utilizan la misma fuente? Habida cuenta de que el error es compartido a la vez por Plinio y Eliano, cabe deducir que estaba ya en su fuente común, y que surgió por una defectuosa interpretación del giro $\pi \rho \dot{\zeta}$ Tò $\pi v \varepsilon \tilde{u} \mu \alpha$ que aparece en Aristóteles, con el significado propio y natural "contra el viento», y que la fuente de Eliano y Plinio debió entender como "a favor del viento», ya que прó con acusativo a menudo significa 'de acuerdo con, con el acompañamiento de'. 
6. Aristóteles, $H A 613$ b 6 ss., afirma que la perdiz pone los huevos e incuba en el mismo nido, y, en cambio, Eliano y Plinio aseguran, por el contrario, que pone los huevos en un nido y que luego los traslada a otro para incubarlos.

He aquí los textos respectivos.

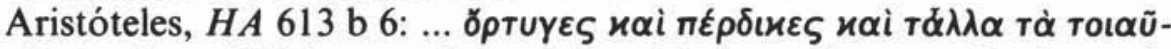

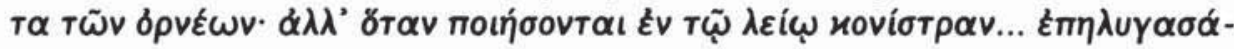

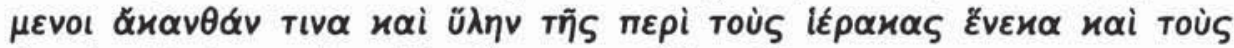

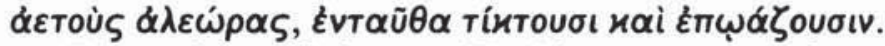

En cambio, Plinio, NH X 100, se expresa asi: Perdices spina et frutice sic muniunt receptaculum ut contra feras abunde uallerentur; ouis stragulum molle puluere contumulant, nec in quo loco peperere incubant; ne cui frequentior conuersatio suspecta sit, transferunt alio.

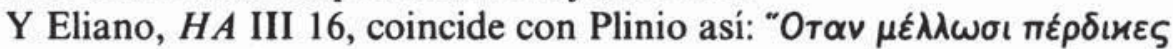

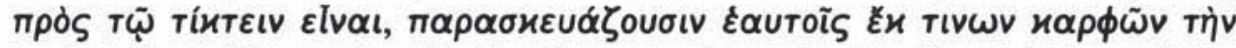

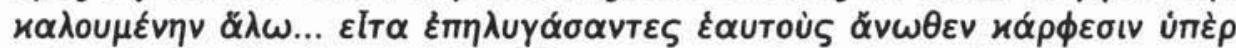

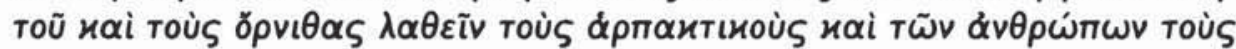

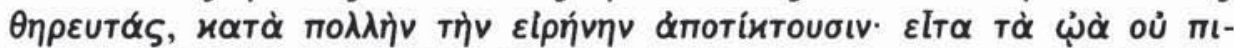

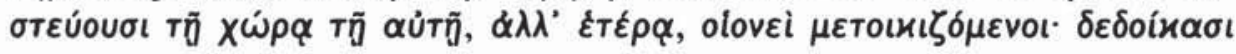

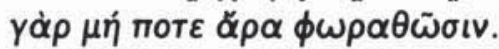

Aunque es posible que Eliano y Plinio dependan en este pasaje de Aristóteles, también cabe que los tres procedan de una fuente común, interpretada correctamente por Aristóteles y erróneamente por los otros dos. Sin embargo, nosotros entendemos que el error de Eliano y Plinio se explica convenientemente con la hipótesis de su dependencia de Aristóteles.

A nuestro juicio ocurrió esto: la frase de Aristóteles, HA 613 b 15-16

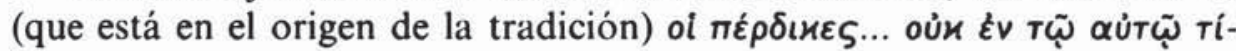

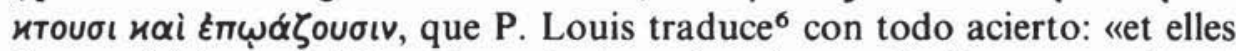
(les perdrix) ne choisissent pas toujours le même endroit pour pondre et couver», fue interpretada erróneamente por Eliano y Plinio, quienes la entendieron así: «las perdices no ponen los huevos en el mismo sitio que incuban". Esto es, el $\varkappa \alpha l$ de la frase de Aristóteles ol $\pi \varepsilon \rho \delta เ и \varepsilon \varsigma \ldots$ oủ $\varepsilon \dot{v} \tau \tau \tilde{\varphi}$

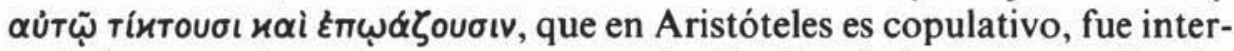
pretado por estos autores como segundo término de la comparación dependiente de $\varepsilon^{2} v \tau \tilde{\varphi} \alpha \alpha \dot{u} T \tilde{\varphi}$.

7. De la manera en que se retira un león descubierto por los cazadores hablan en iguales términos Aristóteles y Eliano. Y la estricta correspondencia entre ambos demuestra que Eliano ha tomado el texto de Aristóteles.

6 Cf. Aristóteles, HA 613 b 15-16, en Aristote. Histoire des animaux, livres VIII-X, París 1969 , p. 80, editados y traducidos por P. Louis. 


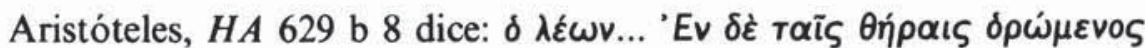

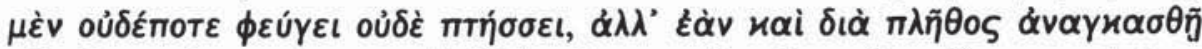

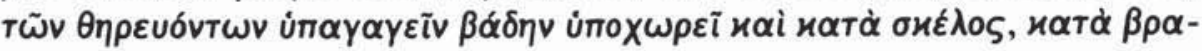
Хù

Pero Eliano, $H A$ IV 34, por el contrario, en idéntico contexto dice lo

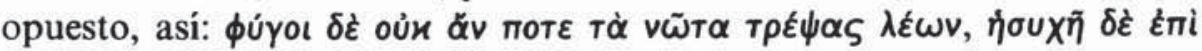

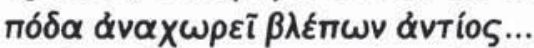

Es evidente, como la coincidencia de todo el contexto demuestra, que Eliano utiliza en última instancia el texto de Aristóteles, lo que pone de manifiesto que hay una frase de Aristóteles a la que Eliano le hace decir algo muy distinto del sentido que el Estagirita le asignó. La frase en cues-

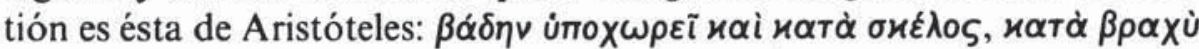

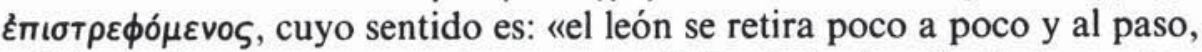
volviendo la cabeza a menudo» o, como $P$. Louis traduce ${ }^{7}$, «il s'en va lentement, sans forcer le pas, en se détournant par instants». Pero Eliano mo-

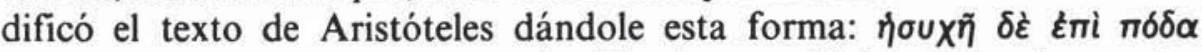

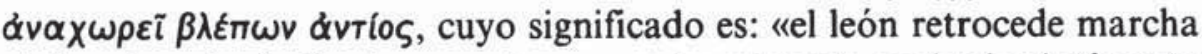
atrás mirando de frente», y así es como lo entiende cualquier intérprete de Eliano como Hercher ${ }^{8}$ con estas palabras: «Numquam terga vertit in fugiendo, sed sensim pedetentimque se recipit, contra intuens».

8. Eliano y Plutarco, utilizando al efecto la misma fuente, interpretan de manera harto distinta la manera en que la osa se adentra en su osera a pasar el letargo invernal.

Plutarco, De sollertia animalium, 971 D-E, dice de ese asunto: $\left(\eta \dot{\gamma} \delta^{\circ}\right.$

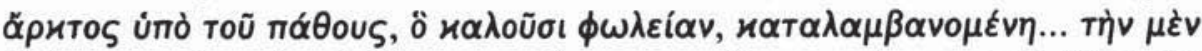

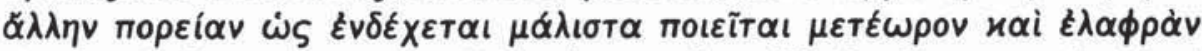

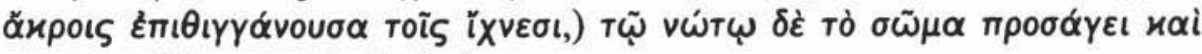

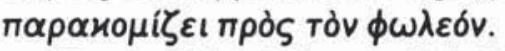

En cambio, Eliano, $H A$ VI 3, describe el mismo hecho de esta otra

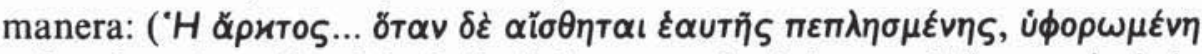

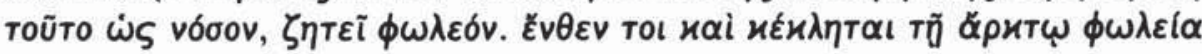

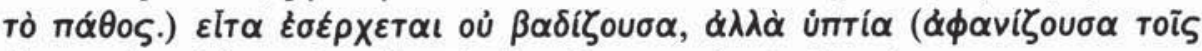

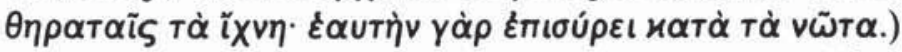

Estos autores difieren en este punto concreto: Plutarco expresamente

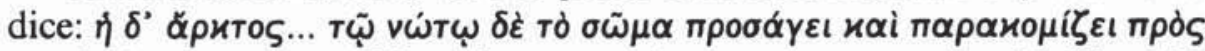
Tòv $\phi \omega \lambda \varepsilon \delta ́ v$, cuyo sentido es interpretado correctamente por H. Cherniss

7 Cf. op. cit., p. 132.

8 R. Hercher, Aeliani de natura animalium, París 1858, p. 64. 
y W. C. Helmbold en su traducción para la colección Loeb, así, en una frase cuyo sujeto es la osa: "turns around and backs into the den".

Pero Eliano cambia estas palabras de Plutarco o de la fuente común

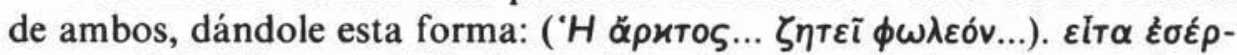

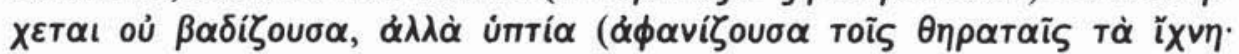

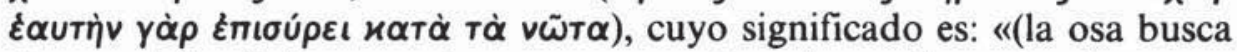
el escondrijo...). Luego entra, pero no caminando con las patas sino tumbada patas arriba (para no dejar huella a los cazadores, pues se arrastra contra las espaldas)".

Que la osa entre en su escondrijo «marcha atrás», $\tau \tilde{\varphi} v \omega \dot{v} \tau \omega$, según refiere Plutarco, es menos espectacular que como concibe esta acción Eliano, y además tiene paralelos. En efecto, el propio Eliano, $H A$ V 53, Plinio, $N H$ VIII 95, y Amiano Marcelino, XXII 15, 22, aseguran que el hipopótamo egipcio devora las mieses de las riberas del Nilo caminando «marcha atrás», teniendo a su espalda el río y delante a los hombres supuestamente perseguidores, para así divisarlos de lejos y poder rehuirlos.

9. Eliano dice que los ciervos del Helesponto tienen una hendidura en una de las dos orejas solamente, y, en cambio, Aristóteles y Plinio afirman que la tienen en las dos orejas.

He aquí los textos respectivos.

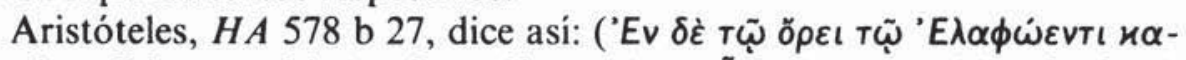

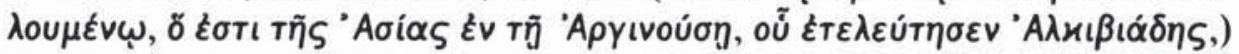

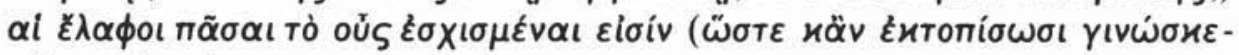
$\sigma \theta \alpha \iota$ тои́тफ.).

También Plinio, $\mathrm{NH}$ VIII 225, de acuerdo con Aristóteles se expresa en los siguientes términos, así: (in Hellesponto in alienos fines non commeant cerui, et circa Arginussam Elaphum montem non excedunt,) auribus etiam in monte fissis.

Pero Eliano, $H A$ VI 13, por el contrario, dice algo muy distinto: $x \alpha i$

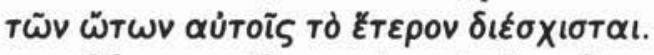

¿Cómo explicar esta extraña afirmación de Eliano? Seguramente asi: es evidente que Aristóteles se refiere al hecho en cuestión usando el singular colectivo tò oús, implicando con él las dos orejas, igual que se refiere a las dos orejas del hombre también con el singular colectivo en $H A 492$

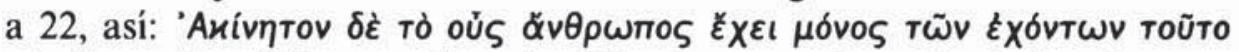
тò $\mu$ ópıov: Pero Eliano no lo entendió así, o no quiso entenderlo, sino que lo interpretó literalmente, como singular puro, y eso explica su particular sentido del texto.

10. Plinio, $N H$ II 107, dice que el antílope egipcio, a la salida del sol, se vuelve de frente a él y lo adora mirándolo de cara, con estas exactas 
palabras: (orygem appellat Aegyptus feram quam in exortu eius) contra stare et contueri (tradit ac uelut adorare, cum sternuerit.)

En cambio, Eliano, $H A \times 28$, afirma que este animal en iguales circunstancias «le vuelve la espalda al sol naciente» y, de esta guisa, «mueve

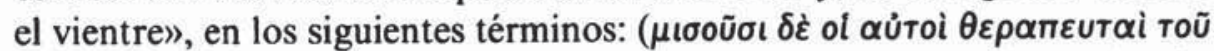

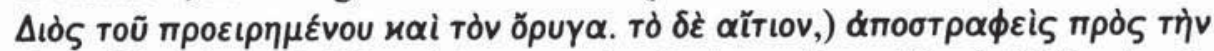

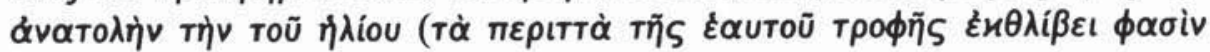
Alyúmtiot).

¿Cómo llegó Eliano a esta extraña deducción pese a utilizar a todas luces la misma fuente que Plinio? Así: es presumible que la fuente común a ambos autores usaba una forma que podía ser $\varepsilon \pi$ de cara a', que Eliano, queriendo o sin querer, entendió como đnобтре́-

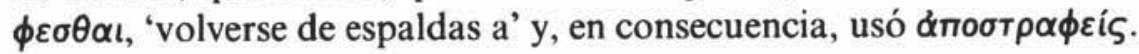

11. Eliano, $H A \times 50$, da la siguiente información relativa a un tal

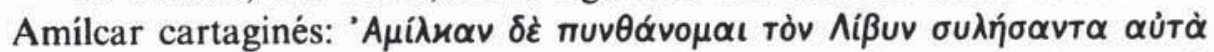

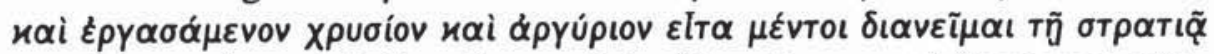

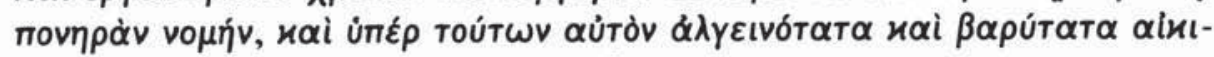

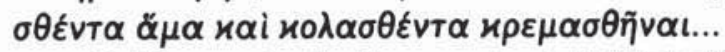

¿Quién es este Amílcar cartaginés? Scholfield ${ }^{9}$ identifica a este Amílcar con el citado por Heródoto, VII 167, y que, según este último autor, fue contemporáneo de Jerjes I y atacó Hímera en 480 a. C.

Pero ninguno de los datos aportados por Eliano confirma el punto de vista de Scholfield, por dos razones, una porque ninguna de las dos tradiciones referentes a la muerte del Amílcar citado por Heródoto (desaparición misteriosa o víctima del fuego del altar) coincide con la señalada por Eliano, y otra, mucho más decisiva, porque la muerte del Amílcar de Eliano fue precedida de "escarnio e ignominia», nada de lo cual afectó al otro Amílcar.

Justamente este dato del "escarnio e ignominia» de que fue víctima el Amílcar de Eliano constituye un criterio definitivo a la hora de identificar a este personaje. En efecto, por ello sabemos que se trata del general cartaginés de ese nombre que en el año 309 a. C. atacó Siracusa y que, fallido su intento, fue hecho prisionero y luego «ultrajado y vejado", para finalmente ser matado, datos estos últimos que coinciden literalmente con los aportados por Eliano.

La fuente fundamental que suministra esta información es Diodoro.

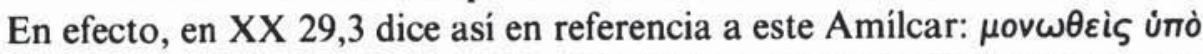

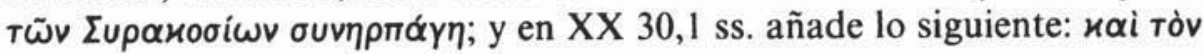

9 Cf. A. F. Scholfield, Aelian. On the characteristics of animals, vol. II, books VIXI, Londres y Cambridge, Massachusetts, 1959, p. 351, nota $b$. 


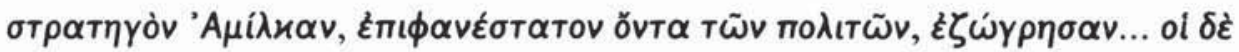

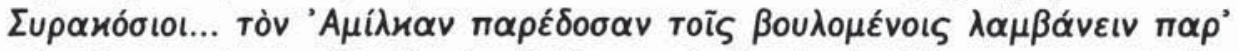

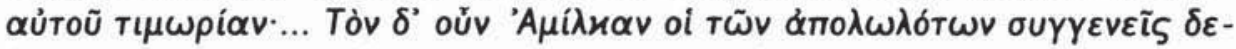

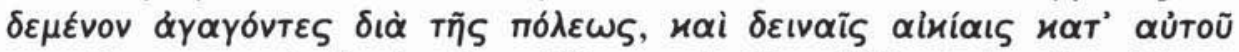

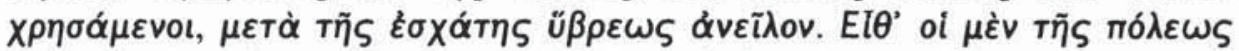

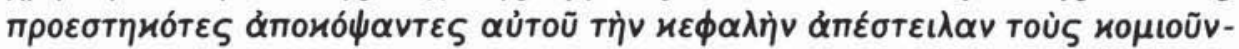

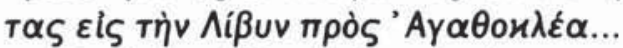

La única diferencia entre las narraciones de Eliano y Diodoro toca solamente a la distinta muerte que cada uno de ellos asigna a este Amílcar: según Diodoro, los siracusanos ávĩ̄ $\lambda$ v a Amílcar, y, en cambio, según Eliano, resulta que Amílcar $и \rho \varepsilon \mu \alpha \sigma \theta \tilde{\eta} v \alpha$.

¿De dónde viene esta diferencia? ¿Es posible dar cuenta de ella? A juzgar por los infinitivos completivos usados por Eliano para referirse a este hecho o a su contexto ( $(\rho \varepsilon \mu \alpha \sigma \theta \tilde{\eta} v \alpha \iota)$ y por Cicerón, De diuinatione I 24,50 (quien al respecto escribe: Apud Agathoclem scriptum in historia est $\mathrm{Ha}$ milcarem Karthaginiensem cum oppugnaret Syracusas, uisum esse audire uocem se... Hamilcaremque ab iis uiuum esse sublatum), parece que los datos relativos a este Amílcar venían expresados en estilo indirecto, esto es, en infinitivo dependiente, justo como aparece en Eliano y Cicerón, de un "se dice", "se cuenta». Este infinitivo, según dan a entender los infinitivos pasados de estos dos autores, debía estar en aoristo, de suerte que podia

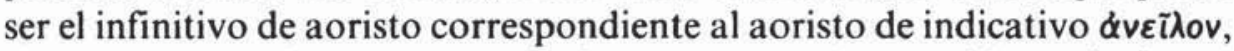
si lo correcto es lo que narra Diodoro, o $к \rho \varepsilon \mu \alpha \sigma \theta \tilde{\eta} v \alpha \iota$, si lo auténtico es la narración de Eliano.

Ahora bien, es evidente, según los datos se encargan de demostrar, que el texto de Eliano sigue al respecto una tradición que le llega de Diodoro.

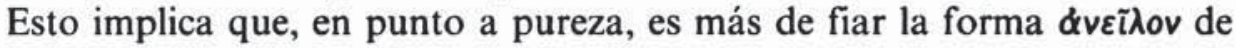
Diodoro que $\varkappa \rho \varepsilon \mu \alpha \sigma \theta \tilde{\eta} v \alpha \iota$ de Eliano. Por lo cual, lo procedente en este caso es dar cuenta del origen de $и \rho \varepsilon \mu \alpha \sigma \theta \tilde{\eta} v \alpha \iota$ y no de $\alpha v \varepsilon i ̃ \lambda o v$.

Para decirlo en pocas palabras: nos parece estar autorizados a sugerir la posibilidad de que la forma $\varkappa \rho \varepsilon \mu \alpha \sigma \theta \tilde{\eta} v \alpha \iota$ de Eliano es el resultado de una interpretación defectuosa de un originario infinitivo de aoristo pasivo

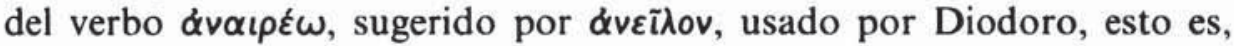
ANAIPEOHNAI. ¿Cómo explicar luego el origen de $и \rho \varepsilon \mu \alpha \sigma \theta \tilde{\eta} v \alpha \iota$ de Eliano? Así: a nuestro juicio, Eliano debió interpretar el infinitivo de Diodoro ANAIPEOHNAI como ANAEPOHNAI, infinitivo de aoristo pasivo de $\alpha v \alpha \varepsilon i \rho \omega$, y por eso Eliano se permitió escribir $и \rho \varepsilon \mu \alpha \sigma \theta \tilde{\eta} v \alpha \iota$, ya que ambos verbos ( $\alpha v \alpha \varepsilon i \rho \omega$ y $и \rho \varepsilon \mu \alpha v v u \mu \iota)$ son de significado parecido y hasta a veces exactamente igual: 'colgar'.

J. VARA 